\title{
Vaccines and Drug-induced Lung Injury
}

Yamamoto $\mathbf{Y}^{*}$

Respiratory Center, Asahikawa Medical University, Asahikawa, Hokkaido, Japan

*Corresponding author: Yamamoto Y, 2-1-1-1, Respiratory Center, Asahikawa Medical University, Midorigaoka-Higashi, Asahikawa, Hokkaido, 078-8510, Japan, Tel: +81-166-69-3290; Fax: +81-166-69-3299; E-mail: yasuyama@asahikawa-med.ac.jp

Received date: Aug 08, 2014; Accepted date: Aug 31, 2015; Published date: Sep 03, 2015

Copyright: (c) 2015 Yamamoto $\mathrm{Y}$, et al. This is an open-access article distributed under the terms of the Creative Commons Attribution License, which permits unrestricted use, distribution, and reproduction in any medium, provided the original author and source are credited.

\section{Short communication}

The corresponding author has no conflict of interest to declare. The author is invited to submit this manuscript in the Short Communication section. Drug-induced lung injuries (DLIs) are adverse drug reactions that specifically occur in the pulmonary system. The main causative agents include cytotoxic drugs, antibiotics, interferon, and anti-rheumatic drugs. More recently, biological reaction modifiers and molecular targeted drugs have emerged as causes of DLIs. Interstitial lung diseases are the most common form of DLIs [1].

Vaccines have rarely been associated with DLIs. One possible reason is that the causal relationship is difficult to prove. This problem is true for vaccines against human papilloma virus (HPV). The author has recently reported a case of interstitial pneumonia that occurred after the vaccination with HPV-16/18 adjuvant system 04 (AS04) vaccines (Cervarix) [2]. Detailed information of this case is available on the respiratory medicine case reports website (http://dx.doi.org/ 10.1016/j.rmcr.2015.06.003).

A middle-aged woman, who had no pre-existing pulmonary diseases, completed three doses of Cervarix. Non-specific interstitial pneumonia developed three months after the last vaccination. A lung biopsy specimen showed lymphocytic alveolitis, providing evidence that cell-mediated immunity likely contributed to the occurrence. The patient had increased levels of serum biomarkers specific to interstitial pneumonias such as Krebs von der Lungen (KL)-6 and surfactant protein (SP)-D. Other causes except the vaccination were eliminated. Of note was that the interstitial pneumonia spontaneously resolved with complete remission of chest radiographic findings and serum biomarkers. The self-limiting course suggested that the interstitial pneumonia occurred with a temporal association with the vaccination. A re-challenge test to Cervarix was not conducted for safety reasons. The interstitial pneumonia was finally diagnosed as a DLI according to the clinical course, chest images, pathological findings, and specific use of Cervarix. Assuming that all drugs are capable of causing a lung injury is the first step for diagnosing DLIs [1]. Vaccines are not exceptions in that DLIs can develop even after the treatment has been completed [1]. Most cases of vaccine-associated DLIs have been diagnosed by clinical judgments [3-5]. Gold standard tests have not been established for the diagnosis of DLIs; however the likelihood of an adverse reaction can be semi-quantified using the Naranjo algoris. Such algorisms can reduce inter- and intra-individual variations with regard to the assessment [6]. Chest imaging findings are non-specific but useful for early diagnosis.[1] Measurements of KL-6 and SP-D may play a supplementary role in the diagnosis of DLIs [1,7]. An exvivo drug stimulation test using peripheral lymphocytes has a quite limited diagnostic value [7]. Further studies are required to develop diagnostic procedures, which are more sensitive and specific to a drug adverse reaction.
Influenza vaccines can cause several types of DLIs, including acute respiratory distress syndrome [3-5]. Watanabe et al. reviewed 7 cases (4 males and 3 females) of DLIs secondary to the influenza vaccination [5]. The median age of onset was relatively high (59 years). Previous pulmonary diseases were present in four cases. All patients had acute symptoms. The time to onset was 1 to 10 days. They all had severe clinical manifestations, but recovered after receiving corticosteroid therapy. Of note, 6 of the 7 cases were Asians [5]. Genetic and environmental factors may affect difference in the susceptibility to DLIs [7].

Unlike influenza vaccines, Cervarix caused a mild and subclinical form of DLI. How did Cervarix affect the pulmonary system? In the disease process of DLIs, drugs can act as a hapten, interact with immune receptors, and trigger danger signals [7]. These actions probably underlie the onset of immune-mediated DLIs [1,7]. These processes were probably attributed to the Cervarix-associated DLI. As a reason, the pathologically proven lymphocytic alveolitis was suggestive of cell-mediated immune responses [2]. Of the constituents of Cervarix, the AS04 adjuvant appeared to be most responsible owing to its strong immunogenicity [8].

Large-scale analyses have not shown that AS04-adjuvanted vaccines increase risks for developing autoimmune disorders $[9,10]$. This trend still remains significant when the subjects are stratified by age [10]. However, the immune-mediated diseases assessed are confined to gastrointestinal, metabolic, musculoskeletal, neuroinflammatory, and skin disorders [10]. Any lung disorders including interstitial pneumonia are not listed. Further studies are needed to clarify the prevalence, outcomes, and risk factors for the Cervarix-associated DLI.

\section{References}

1. Kubo K, Azuma A, Kanazawa M, Kameda H, Kusumoto M, et al. (2013) Consensus statement for the diagnosis and treatment of drug-induced lung injuries. Respir Investig 51:260-277.

2. Yamamoto Y, Kazebayashi Y, Hirai N, Sasaki T, Ohsaki Y (2015) Interstitial lung disease associated with human papillomavirus vaccination. Respiratory Medicine Case Reports 16:15-17.

3. Johnston SD, Kempston A, Robinson TJ (1998) Pneumonitis secondary to the influenza vaccine. Postgrad Med J 74:541-542.

4. Bhurayanontachai R. (2010) Possible life-threatening adverse reaction to monovalent H1N1 vaccine. Crit Care 14: 422.

5. Watanabe S, Waseda Y, Takato H, Inuzuka K, Katayama N, et al. (2013) Influenza vaccine-induced interstitial lung disease. Eur Respir J 41: 474-477.

6. Naranjo CA, Busto U, Sellers EM, Sandor P, Ruiz I, et al. (1981) A method for estimating the probability of adverse drug reactions. Clin Pharmacol Ther 30: 239-245.

7. Matsuno O (2012) Drug-induced interstitial lung disease: Mechanisms and best diagnostic approaches. Respir Res 13: 39. 
Page 2 of 2

8. Didierlaurent AM, Morel S, Lockman L, Giannini SL, Bisteau M, et al (2009) AS04, an aluminum salt- and TLR4 agonist-based adjuvant system, induces a transient localized innate immune response leading to enhanced adaptive immunity. J Immunol 183:6186-6197.

9. Verstraeten T, Descamps D, David MP, Zahaf T, Hardt K, et al. (2008) Analysis of adverse events of potential autoimmune aetiology in a large integrated safety database of AS04 adjuvanted vaccines. Vaccine 26 : 6630-6638.

10. Angelo MG, David MP, Zima J, Baril L, Dubin G, et al. (2014) Pooled analysis of large and long-term safety data from the human papillomavirus-16/18-AS04-adjuvanted vaccine clinical trial programme. Pharmacoepidemiol Drug Saf 23: 466-479. 